\title{
Physical Frailty in COPD Patients with Chronic Respiratory Failure
}

\author{
Sarah Gephine $\mathbb{D}^{1,2}$ \\ Patrick Mucci $\mathbb{D}^{1}$ \\ Jean-Marie Grosbois ${ }^{3}$ \\ François Maltais $\mathbb{D}^{2}$ \\ Didier Saey ${ }^{2}$
}

'University Lille, University Artois, University Littoral Côte d'Opale, ULR 7369 - URePSSS - Unité de Recherche Pluridisciplinaire Sport Santé Société, Lille, F-59000, France; ${ }^{2}$ Centre de Recherche, Institut Universitaire de Cardiologie et de Pneumologie de Québec, Université Laval, Québec, Canada; ${ }^{3}$ Formaction Santé, Pérenchies, France
Correspondence: Didier Saey

Institut Universitaire de Cardiologie et de Pneumologie de Québec, Université Laval, 2725 Chemin Sainte-Foy, Québec, QC, GIV 4G5 Canada

Email Didier.saey@rea.ulaval.ca
Background: The prevalence of physical frailty and its clinical characteristics in advanced chronic obstructive pulmonary disease (COPD) is unknown, as well as the usefulness of functional capacity tests to screen for physical frailty. The aim of the study was to evaluate the proportion and clinical portrait of COPD patients with chronic respiratory failure exhibiting physical frailty at the time of referral to home-based pulmonary rehabilitation. We also evaluate the usefulness of the short physical performance battery (SPPB) and timedup and go (TUG) as potential screening tools for physical frailty. Finally, we evaluated the specific contribution of gait speed to the frailty Fried total score.

Methods: This was a prospective observational study in which physical frailty was defined using Fried criteria (body mass loss, exhaustion, low physical activity, slower walking and weakness). Clinical portrait was documented from daily physical activity, exercise tolerance, functional capacity, anxiety and depressive symptoms, health-related quality of life, and fatigue scores. The ability of the SPPB and TUG to predict physical frailty was investigated using receiver operating characteristic curves. Contribution of each Fried criteria was evaluated with a principal component analysis (PCA).

Results: Amongst the 44 included participants $\left(\mathrm{FEV}_{1}, 33 \pm 13 \%\right.$ of predicted), 19 were physically frail. Frail individuals had lower daily steps number, exercise tolerance and functional capacity, and higher fatigue, anxiety, and depressive symptom scores $(p<0.05)$ compared to non-frail individuals. SPPB and TUG did not have an acceptable detection accuracy for screening physical frailty. PCA indicated that gait speed was the main contributor to the Fried total score of physical frailty.

Conclusion: Physical frailty affects a large proportion of COPD patients with chronic respiratory failure starting a home-based intervention and was associated with worse clinical status. Although the present results need to be confirmed by adequately powered studies, gait speed seems to have the potential to become a simple screening tool for physical frailty in this population.

Keywords: frailty, chronic respiratory failure, pulmonary rehabilitation, gait speed

\section{Introduction}

In advanced chronic obstructive pulmonary disease (COPD), many patients develop hypoxemic or hypercapnic chronic respiratory failure that require long-term oxygen therapy (LTOT) and/or non-invasive ventilation (NIV). ${ }^{1}$ Health-related quality of life (HRQoL), exercise capacity, muscle function, physical activity, mortality are all compromised in COPD patients with chronic respiratory failure, ${ }^{2-4}$ possibly increasing the risk of physical frailty compared to the less severe forms of the disease. 
Physical frailty is a clinical syndrome, first operationalized by Fried phenotypic model, ${ }^{5}$ that characterized individuals with high vulnerability for physical dependency, risk of falls, hospitalizations and death. ${ }^{6}$ Physical frailty affects $20 \%$ of patients with moderate $\mathrm{COPD}^{7}$ and about one-quarter of the patient referred to pulmonary rehabilitation (PR). ${ }^{89}$ Although physical frailty is associated with old age, severe airflow obstruction, dyspnea and frequent exacerbations, ${ }^{7,10}$ the prevalence of physical frailty in the severe forms of COPD has never been documented. Since physical frailty affects the engagement in PR and is associated with a twofold increase in the odds of program noncompletion partly due to the unpredictable health condition, ${ }^{8,11}$ its early detection is important in order to individualize the intervention using additional support and flexible services.

The clinical manifestation of frailty syndrome is well documented in older adults where it is associated with muscle weakness, physical inactivity, disability, risk of falls, malnutrition, and poor HRQoL. ${ }^{12-15}$ Several studies also report that sarcopenia, muscle weakness and malnutrition are highly prevalent in frail patients with COPD., ${ }^{9,16}$ However, the clinical impact of frailty on functional status, physical activity level and HRQoL of patients with severe COPD is also worth considering. Knowledge of the relationships between physical frailty and these clinical features is important since they might be improved by PR. ${ }^{17-19}$

The time needed for Fried phenotypic assessment may restrict its use in clinical practice. Firstly developed to assess the risk of mobility limitations, falls, and disability in older adults, the short physical performance battery (SPPB) and time-up and go (TUG) tests were also used to detect frailty in older adults ${ }^{20-22}$ and proposed as convenient and simple alternatives to the Fried phenotypic assessment. Furthermore, SPPB has shown a reasonable construct validity for screening physical frailty in adult lung transplant candidates in whom its ability to predict post-transplant mortality may surpass that of the Fried phenotypic model. ${ }^{23}$ Although validated to characterize the functional status of patients with COPD, ${ }^{24,25}$ the usefulness of SPPB and TUG to predict physical frailty in COPD has yet to be documented.

Walking is an important aspect of functional activity, as reflected by the associations between slowness and higher risk of disability, cognitive impairment, institutionalization, falls and mortality in older adults. ${ }^{26}$ Because of its clinical relevance, gait speed is one of the physical frailty criteria, but its specific contribution to Fried total score is unknown.

We first aimed to evaluate the proportion of COPD patients with chronic respiratory failure starting a homebased PR program exhibiting physical frailty, and describe the clinical portrait of frail individuals compared to their non-frail counterparts. We specifically focused on the potential relationship of frailty with physical activity level, anxiety and depressive symptoms and HRQoL. The second objective was to evaluate the usefulness of SPPB and TUG as potential screening tools for physical frailty. Finally, we evaluated the specific contribution of gait speed to the frailty Fried total score.

\section{Methods}

\section{Participants and Study Design}

This prospective observational study was part of a larger longitudinal study aimed at evaluating the long-term effectiveness of 8-week home-based PR on functional capacities and physical frailty in COPD patients with chronic respiratory failure. Participants were referred to the homebased PR by their pulmonologist who was in charge of providing the clinical assessment and certifying the presence of COPD according to the Global Initiative for Chronic Obstructive Lung Disease (GOLD) classification system. Participants were then contacted by the PR team to set the first visit at home. Eligible patients were aged 40 years or above with a diagnosis of COPD as a main disease, they had chronic respiratory failure, defined as the requirement for either LTOT and/or NIV. Participants were excluded if they had a poorly controlled psychiatric illness, neurological sequelae, or any bone and joint diseases preventing physical activity. All the patients included in the larger longitudinal study who met the inclusion criteria previously mentioned were included in the present exploratory study.

The study was conducted in accordance with the Declaration of Helsinki and was approved by the observational research protocol evaluation committee of the French Language Society of Pulmonology (CEPRO, number: 2017-007). All participants signed a written informed consent prior to the start of the program which included their approval to use the collected data for research purposes. The research protocol of the present study included two visits at home. 


\section{Assessments}

Lung function, assessed by spirometry according to standard guidelines, ${ }^{27}$ medication and comorbidity data were collected from the individual's medical record provided by the pulmonologist. The burden of comorbidity was assessed using the Charlson Index calculated without adjusting for age and without including COPD in the individual's score, as previously suggested. ${ }^{28}$ During the initial home visit, anthropometrics, anxiety and depressive symptoms, HRQoL, and fatigue data were collected. The second home visit included evaluations of physical frailty, exercise tolerance and functional capacity assessed in a random order. Each measurement was interspersed with a resting period ensuring that heart rate, pulse oxygen saturation, and effort perception had returned their respective baseline value before starting another measurement. Seven days separated the two visits, during which daily physical activity was measured with a tri-axial accelerometer.

\section{Physical Frailty}

Physical frailty was defined using the Fried phenotype model, ${ }^{5}$ including five criteria: unintentional body mass loss history $\geq 4.5 \mathrm{~kg}$, self-reported exhaustion, weekly self-reported energy expenditure using the modified Minnesota Leisure-Time Physical Activity Questionnaire (low physical activity), 4-meter gait speed test (4MGS, slowness) and handgrip dynamometry (weakness). Patients with $\geq 3$ criteria present were considered frail; those with one or two criteria were defined pre-frail, and those with no criteria were considered as robust.

\section{Physical Activity}

In addition to the Minnesota Questionnaire, daily physical activity was objectively assessed using tri-axial Actigraph GT9X accelerometer (Actigraph LLC, Pensacola, FL, USA), previously validated and explicitly recommended for patients with COPD. ${ }^{29,30}$ Participants were instructed to wear the activity monitor on the right hip, during waking hours and over seven consecutive days before the start of the home-based intervention. Physical activity was defined by i) the daily amount of time participant would spend performing sedentary, light, moderate and moderate to vigorous physical activity (MVPA), expressed in minutes and percentage of total recording time and, ii) the daily step number. PA measurement was considered valid only if the patients had a wear-time of $\geq 10 \mathrm{~h}$ per day for at least five of the seven measuring days. ${ }^{30}$ For valid measurements, all available measuring days with a weartime of $\geq 10 \mathrm{~h}$ were taken into account in the analysis. ${ }^{30}$

\section{Exercise Tolerance}

The 6-min stepper test (6MST) validated in COPD was used to evaluate the exercise tolerance at home, as previously described. ${ }^{31}$ Individuals were all familiarized with the stepper prior to the test. Standardized instructions were given, advising the participant to make the maximum number of steps (defined as a single complete movement of raising one foot and putting it down) possible over a six-minute period. No encouragement was given during the test.

\section{Functional Capacity}

Functional capacity was assessed with the SPPB and TUG tests. SPPB is composed of a balance test, 4-meter gait speed (4MGS), and the 5-sit-to-stand repetition test (5STS), which were performed according to the National Institute on Aging protocol. ${ }^{32}$ The standing balance test required participants to maintain each of three stances for 10 seconds; feet placed side-by-side, semi-tandem, and in tandem. The 4MGS measured the time needed to walk 4 meters at a habitual pace. The faster time of two trials was selected and reported in $\mathrm{m} /$ s. ${ }^{33}$ The 5STS required participants to rise from a chair with their arms across their chest, five times. The sum of the three components (each subscore ranged from 0-4) determined the final SPPB score, with a possible range from 0 (functional impairment) to 12 (maximal functional capacity). TUG required the participant to rise from a seated position, walk three meters as quickly and as safely as possible, turn around, walk back, and sit down in the shortest time possible as previously described. $^{34}$

\section{Anxiety and Depressive Symptoms, HRQoL, and Fatigue}

The hospital anxiety and depression (HAD) scale composed of 14 items (7 for anxiety and 7 for depressive symptoms; each subscore can vary from 0 to 21 ; lower scores indicate fewer symptoms) was used to evaluate the anxiety and depression symptoms. ${ }^{35}$ HRQoL was evaluated with the clinical COPD questionnaire (CCQ) which consists of ten questions related to symptoms, mental state, and functional state. ${ }^{36}$ The total score is between 0 and 6 (lower score indicates a greater HRQoL). Fatigue was measured with the fatigue assessment scale (FAS) consisting of 10-item self-report questionnaire. ${ }^{37}$ The response 
scale is a 5-point Likert scale with a total score ranging from 10 to 50 (lower score indicates fewer fatigue).

\section{Data and Statistical Analysis} Proportion and Clinical Features of Physical Frailty

The proportion of individuals meeting the Fried definition of physical frailty is reported as number (\%). Comparisons of baseline variables between frail and non-frail individuals were performed using $t$-tests for the normally distributed quantitative variables while non-parametric Mann-Whitney tests were used for the non-normally distributed variables. Univariate Pearson analyses were performed to evaluate the potential relationship of frailty with, clinical characteristics (age, sex, BMI, forced expiratory volume in $1 \mathrm{~s}\left[\mathrm{FEV}_{1}\right]$, number of exacerbations, mMRC dyspnea scale, Charlson index, medication and nutritional supplements), and study outcomes. Strength of the correlations was categorized according to the correlation coefficient as weak ( 0 to 0.3 ), moderate ( $>0.4$ to 0.6 ), or strong $(>0.7$ to 0.9$){ }^{38}$

\section{Functional Capacity Tests to Detect Physical Frailty}

Receiver operating characteristic (ROC) curves were created to analyze the sensitivity, specificity, area under the curve (AUC) and the positive and negative likelihood ratio (LR) of SPPB and TUG cutoff points to detect physical frailty. An acceptable detection accuracy of the test was defined by an AUC between 0.7 and 0.8, while the detection accuracy of the test was excellent when the AUC > 0.8. ${ }^{39}$ The odds ratios of various cutoff points of these tests to predict physical frailty were also calculated along with their respective confidence intervals.

\section{Specific Contribution of Gait Speed to Physical Frailty}

Documentation of the specific contribution of gait speed to Fried total score is complicated by the potential for collinearity between the two variables, gait speed being one component of the Fried total score. To address this methodological problem, a principal component analysis was performed to determine which of unintentional body mass loss history, self-reported exhaustion, weekly self-reported energy expenditure, 4MGS and handgrip dynamometry are the major contributor to the variance of the Fried total score, in this specific population. This technique permits to reduce the dimensionality of the dataset to few components while minimizing loss of information. Results from the principal component analysis indicate the proportion of the variance explains by each component. On this graphical representation, the closer a variable is to the external boundary the larger is its contribution to the component. We applied the Kaiser criterion to retain two components with eigenvalues equal to or greater than 1 . Factor loadings of $>0.5$ were deemed to be highly relevant to the latent factor.

Statistical analyses were performed using the SPSS statistical software programs, version 19.0 (IBM SPSS, Chicago, IL, USA). Variables were expressed as mean \pm standard deviation or as frequencies and percentages and were tested for normality.

\section{Results}

A total of 55 patients were contacted for possible study participation. Among them, two declines participation and four did not meet the inclusion criteria (COPD was not the main disease). Forty-nine patients gave informed consent to participate in the study; among them, one died, one withdrew consent between the initial and the second visit, and three were excluded because they refused the physical frailty assessment. Anthropometric data, pulmonary function and COPD related-comorbidities of the remaining 44 participants are presented in Table 1. The majority of participants were men, overweight, ex-smokers, with severe airflow limitation and frequent exacerbations (Table 1). Thirty-nine participants were treated with LTOT and NIV and five received only NIV.

\section{Physical Frailty Proportion}

Physical frailty was identified in 19 (43\%) participants; 21 (47\%) participants had one or two Fried criteria and only 4 participants did not meet any of Fried criteria. The latter two categories of participants were grouped under the term "non-frail" participants. Amongst the frail criteria, exhaustion was the most common marker of frailty in each group (total group: 68\%; frail: 89\%; non-frail: 52\%) (Figure 1). No non-frail participant met the 4MGS slowness criteria (Figure 1).

\section{Clinical Features of Physical Frailty}

We did not find significant differences in anthropometric data, pulmonary function or Charlson comorbidity index between the physically frail and non-frail groups (Table 1). The proportion of frail participants on inhaled corticosteroids and nutritional supplements was greater compared to that of the non-frail individuals $(\mathrm{p}<0.05)$. On average, frail individuals had lower exercise tolerance and functional capacity (balance, 4MGS, SPPB total score), and higher 
Table I Anthropometrics, Medical and Pulmonary Function Characteristics

\begin{tabular}{|c|c|c|c|c|}
\hline Characteristics & Total Group $(n=44)$ & Frail $(n=19)$ & Non-Frail $(n=25)$ & p-value \\
\hline Age, years & $66 \pm 8$ & $67 \pm 9$ & $65 \pm 8$ & 0.49 \\
\hline Female, n (\%) & $14(32)$ & $4(2 I)$ & $9(36)$ & 0.29 \\
\hline BMI, $\mathrm{kg} / \mathrm{m}^{2}$ & $26 \pm 7$ & $24 \pm 7$ & $27 \pm 7$ & 0.17 \\
\hline Current smokers, n (\%) & $8(18)$ & $2(10)$ & $6(24)$ & 0.30 \\
\hline $\mathrm{FEV}_{1}, \%$ of predicted & $33 \pm 13$ & $30 \pm 9$ & $36 \pm 16$ & 0.18 \\
\hline $\mathrm{FEV}_{\mathrm{I}} / \mathrm{FVC}, \%$ & $50 \pm 14$ & $48 \pm 8$ & $51 \pm 17$ & 0.52 \\
\hline GOLD stage, n (\%) & & & & 0.35 \\
\hline 2 & $3(7)$ & $0(0)$ & $3(12)$ & \\
\hline 3 & $18(4 \mid)$ & $8(42)$ & $10(40)$ & \\
\hline 4 & $23(52)$ & II (58) & $12(48)$ & \\
\hline Exacerbation $\geq 2$ in last year, $n$ (\%) & $29(66)$ & $13(68)$ & $16(64)$ & 0.77 \\
\hline mMRC dyspnea score & $3.2 \pm 0.9$ & $3.4 \pm 0.9$ & $3.0 \pm 0.9$ & 0.07 \\
\hline LTOT, n (\%) & $39(89)$ & $18(95)$ & $21(84)$ & 0.26 \\
\hline NIV, n (\%) & $29(66)$ & $12(63)$ & $14(56)$ & 0.57 \\
\hline CPAP, n (\%) & $6(14)$ & I (5) & $4(16)$ & 0.28 \\
\hline Short-acting $\beta 2$-agonists, n (\%) & $30(68)$ & $14(74)$ & $16(64)$ & 0.51 \\
\hline Long-acting $\beta 2$-agonists, $\mathrm{n}(\%)$ & $9(20)$ & $3(16)$ & $6(24)$ & 0.52 \\
\hline Long-acting anticholinergic, n (\%) & $43(98)$ & $19(100)$ & $24(96)$ & $0.4 \mathrm{I}$ \\
\hline Bronchodilator with corticosteroids, n (\%) & $16(36)$ & $4(2 I)$ & $12(48)$ & 0.07 \\
\hline Inhaled corticosteroids, n (\%) & $25(57)$ & $14(74)$ & II (44) & 0.04 \\
\hline Oral corticosteroids, n (\%) & $4(9)$ & $2(I I)$ & $2(8)$ & 0.79 \\
\hline Charlson Index & $2.7 \pm 1.9$ & $3.1 \pm 1.9$ & $2.4 \pm 1.9$ & 0.18 \\
\hline Nutritional supplements, n (\%) & II (25) & $9(47)$ & $4(16)$ & 0.03 \\
\hline
\end{tabular}

Notes: Values are presented as mean \pm SD or number (\%). Non-frail group: 0,1 or 2 Fried criteria.

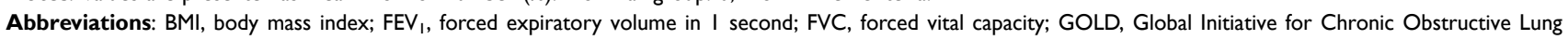
Disease; mMRC, modified Medical Research Council dyspnea scale; LTOT, long-term oxygen therapy; NIV, non-invasive ventilation; CPAP, continuous positive airway pressure.

fatigue scores and anxiety and depressive symptoms $(\mathrm{p}<0.05)$ compared to their non-frail counterparts (Table 2). The CCQ score difference between the two groups did not reach statistical significance $(\mathrm{p}=0.06)$.

Physical activity measurement was obtained in only 33 participants (3 participants refused to wear the activity monitor and in 8 participants, the criteria for a valid measurement were not met). The daily number of steps was significantly different between groups (1392 \pm 1249 versus $2666 \pm 2978$ steps per day, $\mathrm{p}<0.05$, physical frailty vs non-frail); while the daily time spent in sedentary, light and moderate activities was similar between groups $(82 \pm 9 \%$ vs $76 \pm 15 \%, p=0.69$;
$17 \pm 8 \%$ vs $24 \pm 14 \%, \mathrm{p}=0.08 ; 1 \pm 1 \%$ vs $1 \pm 2 \%, \mathrm{p}=0.90$, frail vs non-frail, respectively) (Figure 2).

Univariate analyses are presented in Table 3. Physical frailty was moderately correlated with exercise tolerance, functional capacity, anxiety and depressive symptoms, HRQoL, and fatigue score (Table 3). The correlations with daily physical activity variables were weak.

\section{Functional Capacity Tests to Detect Physical Frailty}

The usefulness of SPPB and TUG to detect physical frailty is illustrated in Figure 3. The AUCs were 0.69 and 0.64, for 


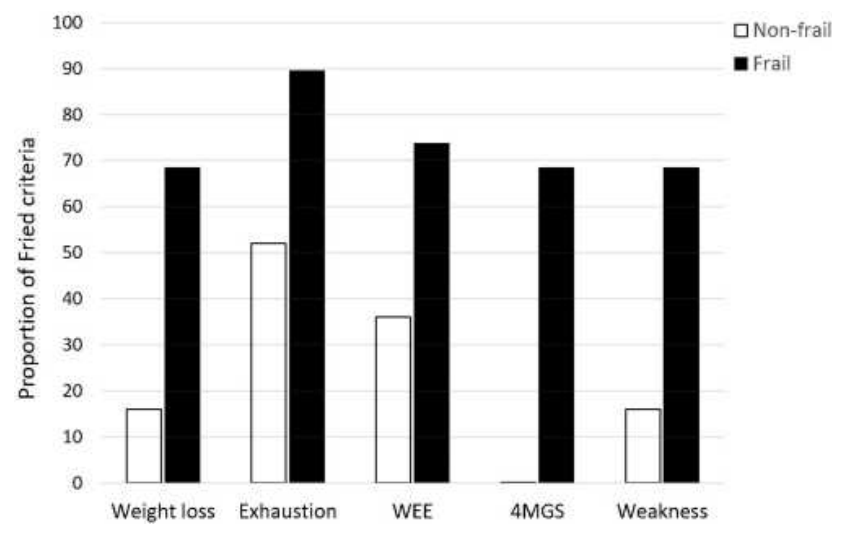

Figure I Prevalence of physical frailty criteria in frail and non-frail individuals. Abbreviations: WEE, weakly energy expenditure estimated from the Minnesota Leisure-Time Physical Activity Questionnaire; 4MGS, 4-meter gait speed.

the SPPB and TUG, respectively. According to the AUC cuts-off, both tests did not have an acceptable detection accuracy for screening physical frailty. Table S1 displays the sensitivity and specificity of SPPB and TUG to screen for physical frailty. According to Figure 3 and Table S1, the best cutoff points for the detection of physical frailty were $\leq$ 10 points and $\geq 8.5$ seconds, for SPPB and TUG, respectively. Moreover, the odds of being physically frail were 2.9-fold (95\% CI $=0.8-11.4)$ higher in individuals with a SPPB score $\leq 10$ points compared to those with SPPB score $>10$ points, and 3.8 -fold $(95 \% \mathrm{CI}=1.1-13.7)$ higher in individuals had a TUG score $\geq 8.5$ seconds compared to those with a TUG score $<8.5$ seconds.

\section{Specific Contribution of Gait Speed to Physical Frailty}

Results of the principal component analysis are represented in Figure 4 and the associated values are displayed in Table $\underline{\mathrm{S} 2}$. The first component explained $31.2 \%$ of the physical frailty score. On the first component, 4MGS had the highest positive loading $(0.67)$, contributing the most to the physical frailty score. The second component explained $26.2 \%$ of the physical frailty score; on this component, weakness had the highest positive loading (0.76) (Table S3). Cumulatively, components 1 and 2 explain $57.4 \%$ of the physical frailty score. The odds ratios of various cutoff points of the $4 \mathrm{MGS}$ to predict physical frailty are presented in Table 4.

\section{Discussion}

Physical frailty affected a large proportion of COPD patients with chronic respiratory failure at the time of starting a home-based PR program. Physically frail participants were using more inhaled corticosteroids and nutritional supplements, and had lower daily steps number, exercise tolerance and functional capacity, and higher anxiety and depressive symptoms, and fatigue scores compared to their non-frail counterparts. Furthermore, physical frailty was moderately correlated with exercise tolerance, functional capacity, anxiety and depressive symptoms, HRQoL, and fatigue scores. These results underscore the challenge of health professionals when caring for patients

Table 2 Clinical Assessments

\begin{tabular}{|c|c|c|c|c|}
\hline Characteristics & Total Group $(n=44)$ & Frail $(n=19)$ & Non-Frail $(n=25)$ & p-value \\
\hline 6MST, strokes & $249 \pm 150$ & $197 \pm 175$ & $288 \pm 116$ & 0.03 \\
\hline \multicolumn{5}{|l|}{ SPPB } \\
\hline Balance, score (0-4) & $3.6 \pm 0.8$ & $3.3 \pm 1.0$ & $3.8 \pm 0.5$ & 0.02 \\
\hline 4MGS, $\mathrm{m} / \mathrm{s}$ & $0.8 \pm 0.2$ & $0.7 \pm 0.2$ & $0.9 \pm 0.1$ & $<0.01$ \\
\hline 5STS, seconds & $12.7 \pm 3.5$ & $12.9 \pm 3.7$ & $12.5 \pm 3.3$ & 0.73 \\
\hline Total score $(0-12)$ & $9.7 \pm 1.9$ & $8.9 \pm 2.1$ & $10.3 \pm 1.4$ & 0.03 \\
\hline TUG, seconds & $9.8 \pm 5.4$ & $9.9 \pm 3.1$ & $9.6 \pm 6.7$ & 0.13 \\
\hline Anxiety symptoms, score & $9.8 \pm 4.8$ & $12.2 \pm 5.2$ & $7.9 \pm 3.8$ & $<0.01$ \\
\hline Depressive symptoms, score & $8.1 \pm 4.1$ & $9.7 \pm 4.6$ & $7.0 \pm 3.5$ & 0.01 \\
\hline CCQ, score & $2.9 \pm 1.3$ & $3.4 \pm 1.5$ & $2.6 \pm 0.9$ & 0.06 \\
\hline FAS, score & $25.6 \pm 7.9$ & $28.9 \pm 8.5$ & $23.1 \pm 6.5$ & 0.01 \\
\hline
\end{tabular}

Note: Values are presented as mean \pm SD.

Abbreviations: 6MST, 6-minute stepper test; SPPB, short physical performance battery test; 4MGS, 4-meter gait speed; 5STS, 5-sit-to-stand test; TUG, timed-up and go test; CCQ, clinical COPD questionnaire; FAS, fatigue assessment scale. 


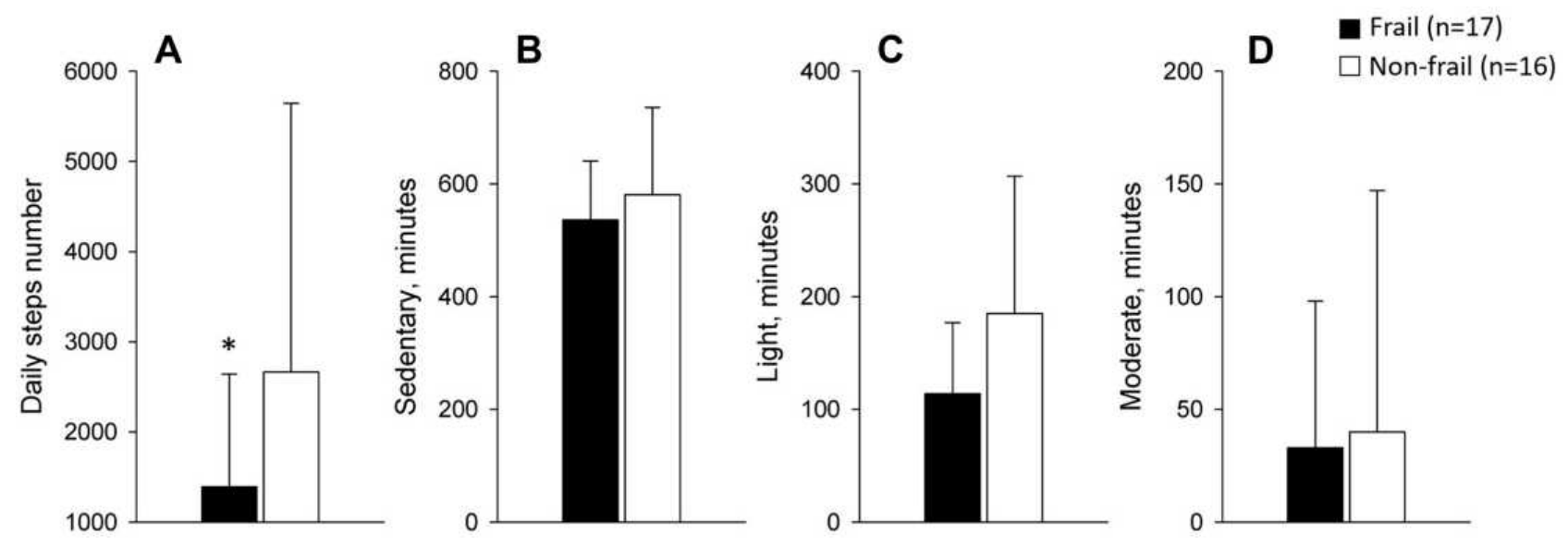

Figure 2 Daily physical activity levels defined by the number of steps $(\mathbf{A})$ and the number of time spent in sedentary (B), light (C) and moderate (D) activities in minutes. Values are mean \pm SD. ${ }^{*} p<0.05$ frail versus non-frail. Since neither group spent any time in vigorous activity, this physical activity parameter is not presented.

with severe COPD, but also highlight the heavy clinical burden COPD patients with chronic respiratory failure have to deal with when starting a PR program.

The second aim of the study was to evaluate the usefulness of SPPB and TUG for screening physical frailty. Our results suggest that neither SPPB nor TUG has an acceptable detection accuracy for screening physical frailty. Because of its clinical relevance, we used a principal component analysis to evaluate the specific contribution of gait speed to frailty score. Results showed

Table 3 Pearson Correlation with Physical Frailty

\begin{tabular}{|l|c|c|}
\hline Characteristics (n=44) & Physical Frailty & p value \\
\hline mMRC dyspnea score & 0.39 & $<0.01$ \\
\hline 6MST, strokes & -0.43 & $<0.01$ \\
\hline SPPB, total score (0-12) & -0.43 & $<0.01$ \\
\hline TUG, seconds & 0.02 & 0.89 \\
\hline Anxiety symptoms, score & 0.45 & $<0.01$ \\
\hline Depressive symptoms, score & 0.40 & $<0.01$ \\
\hline CCQ, score & 0.50 & $<0.01$ \\
\hline FAS, score & 0.52 & $<0.01$ \\
\hline Daily physical activity & & \\
Steps, number & -0.38 & 0.03 \\
Sedentary, minutes & -0.16 & 0.38 \\
Light, minutes & -0.37 & 0.03 \\
Moderate, minutes & -0.08 & 0.67 \\
\hline
\end{tabular}

Abbreviations: mMRC, modified Medical Research Council dyspnea scale; 6MST, 6-minute stepper test; SPPB, short physical performance battery test; TUG, timedup and go test; CCQ, clinical COPD questionnaire; FAS, fatigue assessment scale. that amongst Fried criteria, gait speed was the main contributor to the total score of physical frailty.

Frail status is highly associated with the severity of airflow obstruction and frequency of exacerbations; consequently, patients with severe COPD have an increased risk of frailty compared to those affected by milder forms of the disease. ${ }^{10}$ We found that $43 \%$ and $47 \%$ of the individuals referred for the home PR intervention were physically frail and prefrail, respectively. Taking together, $90 \%$ of our population had at least one Fried criterion, with exhaustion as the most common marker of physical frailty, consistent with a previous report. ${ }^{8}$ This result is higher than previously reported, where 25 to $28 \%$ of patients with moderate to severe COPD were physically frail when starting a center-based PR. ${ }^{8,9}$ This can be explained by the presence of severe airflow limitation and chronic respiratory failure in the present study population. This result highlights the importance of implementing physical frailty assessment in PR settings, especially in people requiring LTOT and NIV.

Age, sex, severity of airway obstruction, body mass index, number of comorbidity or number of exacerbation during the last year, could not discriminate the physical frail individuals from the non-frail ones. These factors were previously associated with frailty in COPD, but when using another definition than Fried phenotype. ${ }^{10,40}$ However, we found that the proportion of physical frail individuals using inhaled corticosteroids and daily nutritional supplements was greater than what was seen in nonfrail ones, consistent with a previous result in which malnutrition was present in $63 \%$ of the physically frail individuals. ${ }^{9}$ Moreover, the physical frail group had 

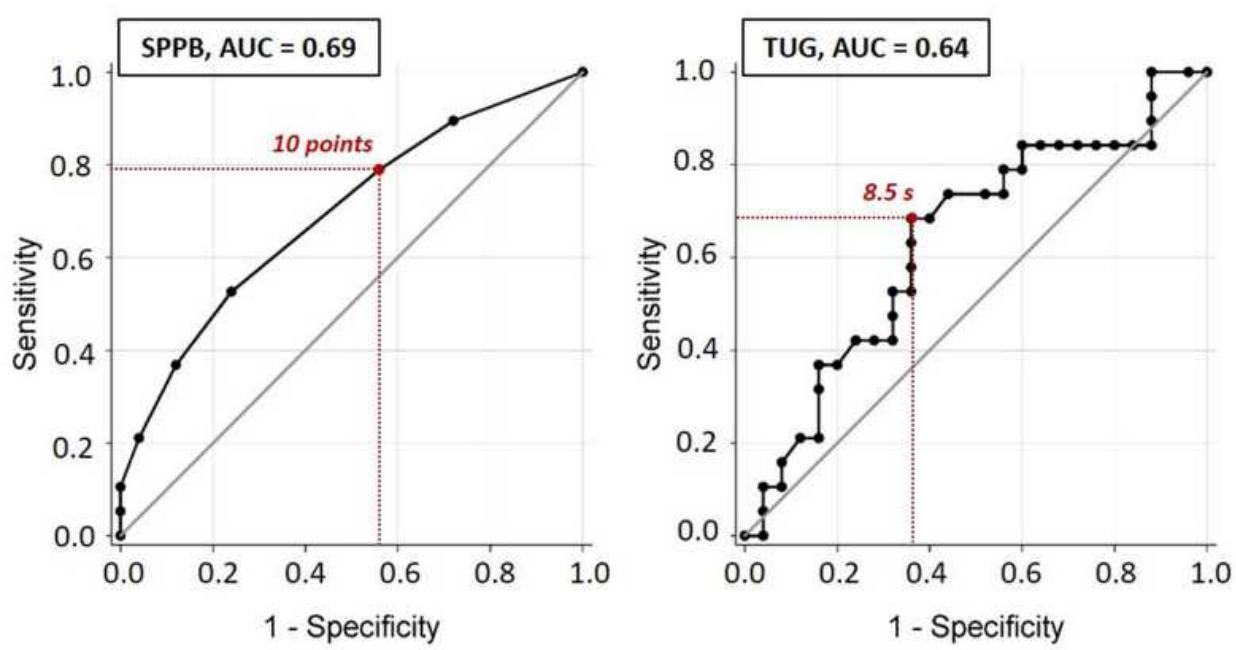

Figure 3 ROC curves of the SPPB and TUG as predictors of physical frailty.

Abbreviations: AUC, area under the curve; SPPB, short physical performance battery test; TUG, timed-up and go test.

lower daily steps number, exercise tolerance and functional capacity compared to the non-frail group.

The Minnesota Questionnaire which is used to document weekly self-reported energy expenditure in the Fried

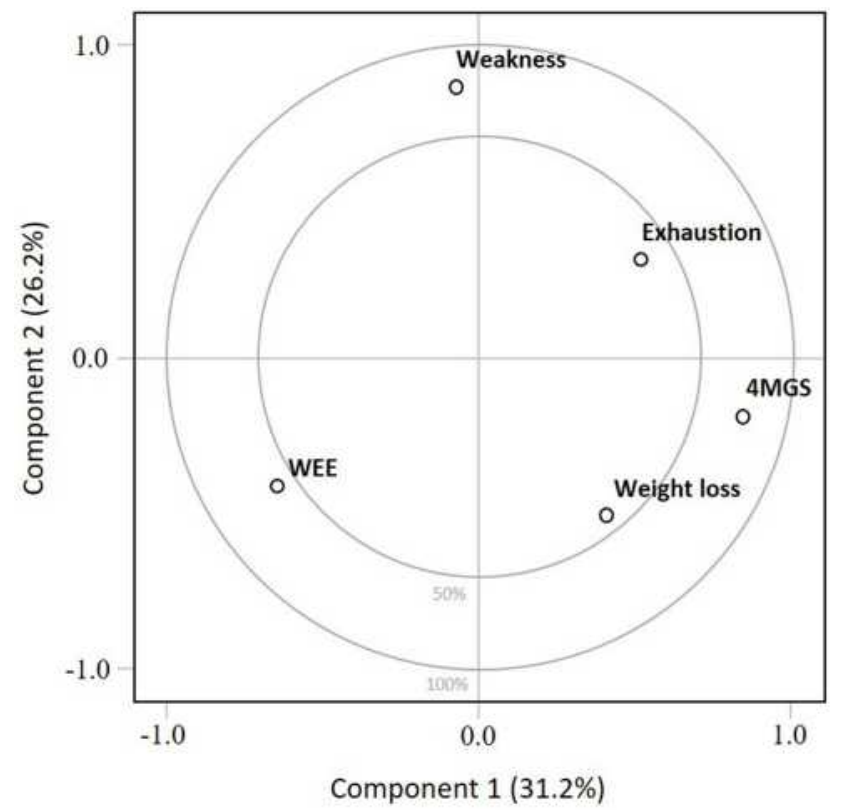

Figure 4 Principal component analysis of the first two principal components of physical frailty. Principal component analysis (PCA) is a simple graphical display used to study the overall structure of the dataset and to obtain a visual understanding of relationships between the included variables (ie, Fried criteria) ${ }^{52}$. On this graphical representation, the closer a variable is to the external boundary the larger is its contribution to the component. PCA analysis indicated that the first component and the second component explained $31.2 \%$ and $26.2 \%$ of the variance of the physical frailty score, respectively. On the first component, 4MGS had the highest positive loading, while on the second component, weakness had the highest positive loading. Cumulatively, components I and 2 explained $57.4 \%$ of the variance of physical frailty score.

Abbreviations: WEE, weekly energy expenditure estimated from the Minnesota Leisure-Time Physical Activity Questionnaire; 4MGS, 4-meter gait speed. physical frailty assessment is time-consuming and requires remembrance of the practice of physical activity over the past year. We used accelerometry to provide an objective assessment of physical activity in this population and found that COPD patients with chronic respiratory failure have a remarkably low level of domestic daily life activities. ${ }^{41}$ We also confirmed the existing relationship between physical frailty and low daily physical activity previously reported in healthy older adults. ${ }^{42}$ Regardless of the physical frailty status, the number of steps per day and the time spent in moderate physical activity were well below the guideline recommendations. ${ }^{43}$

Although under-recognized, fatigue, anxiety and depressive symptoms are commonly reported in patients with COPD starting PR. ${ }^{44,45}$ We confirmed that physically frail patients with COPD present higher fatigue, and anxiety and depressive symptoms compared to the non-frail. ${ }^{8}$ We extend this knowledge by demonstrating moderate correlations between physical frailty and anxiety and depressive symptoms, fatigue and poor HRQoL, in line with the results of previous studies in older adults. ${ }^{15,46,47}$ Moreover, although the CCQ score was not significantly different between the frail and non-frail groups $(p=0.06)$, the positive difference $(+0.8$ for the physically frail patients compared to the non-frail ones) was higher than the minimal clinically important difference for this measure $(0.3$ to 0.5$){ }^{48}$

The concomitant occurrence of physical frailty, physical inactivity, exercise intolerance, general fatigue, anxiety and depressive symptoms, and poor HRQoL might prevent patients with COPD from fully engaging in pulmonary 
Table 4 Odds Ratio for Detecting Physical Frailty

\begin{tabular}{|l|c|c|}
\hline Detection of Physical Frailty & Odds Ratio & $\mathbf{9 5 \%} \mathbf{~ C l}$ \\
\hline SPPB, points & & \\
\hline$\leq 10$ & 2.9 & $0.8-11.4$ \\
\hline TUG, seconds & & \\
\hline$\geq 8.5$ & 3.8 & $1.1-13.7$ \\
\hline 4 MGS, m/s & & \\
\hline$\leq 1$ & 1.3 & $0.3-6.4$ \\
\hline$\leq 0.95$ & 3.0 & $0.7-13.2$ \\
\hline$\leq 0.90$ & 4.2 & $1.0-18.1$ \\
\hline$\leq 0.85$ & 5.6 & $1.4-22.0$ \\
\hline$\leq 0.8$ & 9.6 & $2.4-39.4$ \\
\hline$\leq 0.75$ & 12.6 & $2.7-57.7$ \\
\hline$\leq 0.70$ & 12.8 & $2.3-70.1$ \\
\hline$\leq 0.65$ & 21.6 & $2.4-193.7$ \\
\hline
\end{tabular}

Abbreviations: SPPB, short physical performance battery test; TUG, timed-up and go test; 4MGS, 4-meter gait speed; Cl, confidence interval.

rehabilitation. Indeed, despite that frail patients with COPD respond favorably to $\mathrm{PR},{ }^{8}$ our results confirmed that this population accumulated multidimensional loss that might compromise the effectiveness of traditional PR. ${ }^{11}$ It is thus crucial to identify these people at the time of their engagement in those programs and understand their multidimensional concerns in order to individualise the intervention to suit their needs and priorities. ${ }^{49}$ For example, the exercise training program and the psychologic and motivational support provided during traditional PR could be individualised according to the Fried phenotypic model that may highlight the presence of impaired muscle strength, sedentary lifestyle, walking disability, depressive symptoms, or risk of malnutrition upon which specific therapeutic interventions may be undertaken.

SPPB and TUG tests are commonly used in clinical settings and are validated to assess the risk of mobility limitations, falls and disability in patients with COPD. ${ }^{24,25}$ Moreover, an SPPB score $\leq 8$ and a TUG score $\leq 10$ seconds were selected as a cut-off for screening physical frailty in older adults. ${ }^{20-22}$ In the present study, both tests did not have an acceptable detection accuracy for screening physical frailty according to the AUC value $(<0.70)$.
Finally, our results indicated that gait speed was the main contributor to the Fried total score of physical frailty. This result is further supported by fact that none of the individuals of the non-frail group met the Fried slowness criterion, possibly making the 4MGS a discriminant factor for physical frailty. The utility of the 4MGS has been already highlighted in older adults where a slow walking speed predicts a higher risk of disability, cognitive impairment, institutionalization, falls and mortality. ${ }^{26}$ Moreover, the 4MGS can be easily implemented in clinical or homebased settings, offering a simple and safe surrogate of functional capacity in COPD patients. ${ }^{50}$ Schoon et al showed that a gait speed $\leq 0.8 \mathrm{~m} / \mathrm{s}$ had an excellent diagnostic value for screening for physical frailty in older adults, whether it is assessed with Fried phenotypic model or with Frailty Index tool (not including 4MGS as a component). ${ }^{51}$ Using the same cutoff, the present study demonstrated that in COPD patients with chronic respiratory failure, a gait speed $\leq 0.8 \mathrm{~m} / \mathrm{s}$ was associated with a 10-fold increase in the odds of being physically frail.

\section{Methodological Considerations}

The exploratory nature of this study and the small sample size limit the scope of the present results, which need to be confirmed by an adequately powered study. Moreover, we purposefully selected COPD patients with chronic respiratory failure, thus the present results are not generalizable to patients with milder forms of COPD. In the same way, we selected Fried criteria to define physical frailty which cannot be substituted with other frailty assessments. We also acknowledge that we did not strictly followed the Fried physical frailty categorization by grouping the prefrail and robust patients together. Nevertheless, results were the same when we ran the statistics removing the four robust participants from the non-frail group.

Finally, our intention was not to propose the SPPB, TUG or 4MGS tests as alternatives to the Fried phenotype assessment to diagnose physical frailty but rather to determine a test that would allow for a quick clinical assessment of the participant. This would help to determine whether the patients would require more in-depth physical frailty assessments and if intervention would be necessary.

\section{Conclusion}

Physical frailty affected a large proportion of COPD patients with chronic respiratory failure at the time of starting a home-based PR program and was associated with reduced daily physical activity, exercise intolerance, 
anxiety and depressive symptoms, fatigue and poor functional status, and HRQoL. Although the present results need to be confirmed by adequately powered studies, gait speed seems to have the potential to become a simple screening tool for physical frailty in this population.

\section{Abbreviation}

4MGS, 4-meter gait speed; 5STS, 5-sit-to-stand test; 6MST, 6-minute stepper test; AUC, area under the curve; BMI, body mass index; CCQ, clinical COPD questionnaire; $\mathrm{COPD}$, chronic obstructive pulmonary disease; CPAP, continuous positive airway pressure; FAS, fatigue assessment scale; $\mathrm{FEV}_{1}$, forced expiratory volume in 1 second; FVC, functional vital capacity; GOLD, Global Initiative for Chronic Obstructive Lung Disease; HAD, hospital anxiety and depression scale; HRQoL, health-related quality of life; LTOT, long-term oxygen therapy; mMRC, modified Medical Research Council dyspnea scale; MVPA, moderate to vigorous physical activity; NIV, non-invasive ventilation; PR, pulmonary rehabilitation; ROC, receiver operating characteristic; SPPB, short physical performance battery test; TUG, timed-up and go test; WEE, weakly energy expenditure.

\section{Acknowledgments}

We thank the members of the rehabilitation team: Sophie Duriez, Mathieu Grosbois, Marjorie Lambinet, Gaelle Tywoniuk, Valentine Opsomer, Florence Urbain, and Virginie Wauquier. We also thank Georges Baquet (Univ. Lille, Univ. Artois, Univ. Littoral Côte d'Opale, ULR 7369 - URePSSS F-59000 Lille, France) for his assistance with the accelerometer and Serge Simard (Centre De Recherche, Institut universitaire de cardiologie et de pneumologie de Québec, Université Laval, Québec, Canada) for his assistance with the statistical analysis.

\section{Author Contributions}

All authors made a significant contribution to the work reported, whether that is in the conception, study design, execution, acquisition of data, analysis and interpretation, or in all these areas; took part in drafting, revising or critically reviewing the article; gave final approval of the version to be published; have agreed on the journal to which the article has been submitted; and agree to be accountable for all aspects of the work.

\section{Funding}

Sarah Gephine was supported by doctoral salary from the University of Lille. The delivery of home-based PR was financially supported by Adair, Aeris Santé, Bastide, France Oxygène, Homeperf, LVL, Medopale, NorOx, Santélys, SOS Oxygène, Sysmed, VitalAire, and ARS Hauts-de-France. The funders played no role in the design, conduct or reporting of this study.

\section{Disclosure}

Sarah Gephine reports PhD salary from University of Lille and that the delivery of home-based PR was financially supported by Adair, Aeris Santé, Bastide, France Oxygène, Homeperf, LVL, Medopale, NorOx, Santélys, SOS Oxygène, Sysmed, VitalAire, and ARS Hauts-de-France during the conduct of the study. Jean-Marie Grosbois reports personal fees unrelated to the submitted work from AstraZeneca, Boehringer Ingelheim, Chiesi, GlaxoSmithKlein, Novartis, Vitalaire, and CSL Behring, and that FormAction Santé received financial support for the home-based program from Adair, Aeris Santé, Bastide, France Oxygène, Homeperf, LVL Medical, Medopale, NorOx, Santélys, Santeo, SOS Oxygène, Sysmed, VitalAire and the ARS Hauts de France. François Maltais reports grants from GlaxoSmithKline, AstraZeneca, Sanofi, Novartis, Boehringer Ingelheim, and Grifols, personal fees from GlaxoSmithKline, Boehringer Ingelheim, Grifols, and Novartis, and reports having a financial participation in Oxynov, a company which is developing an oxygen delivery systemoutside the submitted work. The funders played no role in the design, conduct or reporting of this study. The authors report no other potential conflicts of interest in this work.

\section{References}

1. Budweiser S, Jorres RA, Pfeifer M. Treatment of respiratory failure in COPD. Int $J$ Chron Obstruct Pulmon Dis. 2008;3(4):605-618. doi:10.2147/COPD.S3814

2. Carone M, Antoniu S, Baiardi P, et al. Predictors of mortality in patients with COPD and chronic respiratory failure: the quality-oflife evaluation and survival study (QuESS): a Three-Year Study. COPD. 2016;13(2):130-138. doi:10.3109/15412555.2015.1067294

3. Mazzarin C, Kovelis D, Biazim S, Pitta F, Valderramas S. Physical inactivity, functional status and exercise capacity in COPD Patients Receiving Home-Based Oxygen Therapy. COPD. 2018;1-6.

4. Coquart JB, Le Rouzic O, Racil G, Wallaert B, Grosbois JM. Real-life feasibility and effectiveness of home-based pulmonary rehabilitation in chronic obstructive pulmonary disease requiring medical equipment. Int J Chron Obstruct Pulmon Dis. 2017;12:3549-3556. doi:10.2147/ COPD.S150827 
5. Fried LP, Tangen CM, Walston J, et al. Frailty in older adults: evidence for a phenotype. J Gerontol a Biol Sci Med Sci. 2001;56(3):M146M156. doi:10.1093/gerona/56.3.M146

6. Morley JE, Vellas B, van Kan GA, et al. Frailty consensus: a call to action. J Am Med Dir Assoc. 2013;14(6):392-397. doi:10.1016/j. jamda.2013.03.022

7. Marengoni A, Vetrano DL, Manes-Gravina E, Bernabei R, Onder G, Palmer K. The relationship between COPD and frailty: a systematic review and meta-analysis of observational studies. Chest. 2018;154 (1):21-40. doi:10.1016/j.chest.2018.02.014

8. Maddocks M, Kon SS, Canavan JL, et al. Physical frailty and pulmonary rehabilitation in COPD: a prospective cohort study. Thorax. 2016;71(11):988-995. doi:10.1136/thoraxjnl-2016-208460

9. Ter Beek L, van der Vaart H, Wempe JB, et al. Coexistence of malnutrition, frailty, physical frailty and disability in patients with COPD starting a pulmonary rehabilitation program. Clin Nutr. 2020;39(8):2557-2563. doi:10.1016/j.clnu.2019.11.016

10. Lahousse L, Ziere G, Verlinden VJ, et al. Risk of frailty in elderly with COPD: a population-based study. J Gerontol a Biol Sci Med Sci. 2016;71(5):689-695. doi:10.1093/gerona/glv154

11. Brighton LJ, Bristowe K, Bayly J, et al. Experiences of pulmonary rehabilitation in people living with chronic obstructive pulmonary disease and frailty. A qualitative interview study. Ann Am Thorac Soc. 2020;17(10):1213-1221. doi:10.1513/AnnalsATS.201910-800OC

12. Ansai JH, Farche ACS, Rossi PG, de Andrade LP, Nakagawa TH, Takahashi ACM. Performance of different timed up and go subtasks in frailty syndrome. $J$ Geriatr Phys Ther. 2019;42(4):287-293. doi:10.1519/JPT.0000000000000162

13. de la Rica-escuin M, Gonzalez-Vaca J, Varela-Perez R, et al. Frailty and mortality or incident disability in institutionalized older adults: the FINAL study. Maturitas. 2014;78(4):329-334. doi:10.1016/j. maturitas.2014.05.022

14. Laur CV, McNicholl T, Valaitis R, Keller HH. Malnutrition or frailty? Overlap and evidence gaps in the diagnosis and treatment of frailty and malnutrition. Appl Physiol Nutr Metab. 2017;42(5):449-458. doi:10.1139/apnm-2016-0652

15. Crocker TF, Brown L, Clegg A, et al. Quality of life is substantially worse for community-dwelling older people living with frailty: systematic review and meta-analysis. Qual Life Res. 2019;28 (8):2041-2056. doi:10.1007/s11136-019-02149-1

16. Bone AE, Hepgul N, Kon S, Maddocks M. Sarcopenia and frailty in chronic respiratory disease. Chron Respir Dis. 2017;14(1):85-99. doi:10.1177/1479972316679664

17. Beauchamp MK, O’Hoski S, Goldstein RS, Brooks D. Effect of pulmonary rehabilitation on balance in persons with chronic obstructive pulmonary disease. Arch Phys Med Rehabil. 2010;91 (9):1460-1465. doi:10.1016/j.apmr.2010.06.021

18. Spruit MA, Pitta F, McAuley E, ZuWallack RL, Nici L. Pulmonary rehabilitation and physical activity in patients with chronic obstructive pulmonary disease. Am J Respir Crit Care Med. 2015;192 (8):924-933. doi:10.1164/rccm.201505-0929CI

19. McCarthy B, Casey D, Devane D, Murphy K, Murphy E, Lacasse Y. Pulmonary rehabilitation for chronic obstructive pulmonary disease. Cochrane Database Syst Rev. 2015;1(2):CD003793.

20. Perracini MR, Mello M, de Oliveira Maximo R, et al. Diagnostic accuracy of the short physical performance battery for detecting frailty in older people. Phys Ther. 2020;100(1):90-98. doi:10.1093/ ptj/pzz154

21. Ramirez-Velez R, Lopez Saez de Asteasu M, Morley JE, CanoGutierrez CA, Izquierdo M. Performance of the short physical performance battery in identifying the frailty phenotype and predicting geriatric syndromes in community-dwelling elderly $J$ Nutr Health Aging. 2021;25(2):209-217. doi:10.1007/s12603020-1484-3
22. Savva GM, Donoghue OA, Horgan F, O'Regan C, Cronin H, Kenny RA. Using timed up-and-go to identify frail members of the older population. J Gerontol a Biol Sci Med Sci. 2013;68(4):441-446. doi:10.1093/gerona/gls190

23. Singer JP, Diamond JM, Gries CJ, et al. Frailty Phenotypes, Disability, and Outcomes in Adult Candidates for Lung Transplantation. Am J Respir Crit Care Med. 2015;192 (11):1325-1334. doi:10.1164/rccm.201506-1150OC

24. Bernabeu-Mora R, Medina-Mirapeix F, Llamazares-Herran E, Garcia-Guillamon G, Gimenez-Gimenez LM, Sanchez-Nieto JM. The short physical performance battery is a discriminative tool for identifying patients with COPD at risk of disability. Int J Chron Obstruct Pulmon Dis. 2015;10:2619-2626. doi:10.2147/COPD. S94377

25. Reynaud V, Muti D, Pereira B, et al. A TUG Value Longer Than 11 s Predicts Fall Risk at 6-Month in Individuals with COPD. $J$ Clin Med. 2019;8(10):1752. doi:10.3390/jcm8101752

26. Abellan van Kan G, Rolland Y, Andrieu S, et al. Gait speed at usual pace as a predictor of adverse outcomes in community-dwelling older people an International Academy on Nutrition and Aging (IANA) Task Force. J Nutr Health Aging. 2009;13(10):881-889. doi:10.1007/ s12603-009-0246-z

27. American Thoracic Society. Standardization of spirometry. Am $J$ Respir Crit Care Med. 1994;152:1107-1136.

28. Higashimoto Y, Yamagata T, Maeda K, et al. Influence of comorbidities on the efficacy of pulmonary rehabilitation in patients with chronic obstructive pulmonary disease. Geriatr Gerontol Int. 2016;16(8):934-941. doi:10.1111/ggi.12575

29. Rabinovich RA, Louvaris Z, Raste Y, et al. Validity of physical activity monitors during daily life in patients with COPD. Eur Respir J. 2013;42(5):1205-1215. doi:10.1183/09031936.00134312

30. Van Remoortel H, Raste Y, Louvaris Z, et al. Validity of six activity monitors in chronic obstructive pulmonary disease: a comparison with indirect calorimetry. PLoS One. 2012;7(6):e39198. doi:10.1371/journal.pone.0039198

31. Grosbois JM, Riquier C, Chehere B, et al. Six-minute stepper test: a valid clinical exercise tolerance test for COPD patients. Int J Chron Obstruct Pulmon Dis. 2016;11:657-663. doi:10.2147/ COPD.S98635

32. Guralnik JM, Simonsick EM, Ferrucci L, et al. A short physical performance battery assessing lower extremity function: association with self-reported disability and prediction of mortality and nursing home admission. J Gerontol. 1994;49(2):M85-M94. doi:10.1093/geronj/49.2.M85

33. Kon SS, Patel MS, Canavan JL, et al. Reliability and validity of 4-metre gait speed in COPD. Eur Respir J. 2013;42(2):333-340. doi:10.1183/09031936.00162712

34. Mesquita R, Wilke S, Smid DE, et al. Measurement properties of the Timed Up \& Go test in patients with COPD. Chron Respir Dis. 2016;13(4):344-352. doi:10.1177/1479972316647178

35. Lepine JP, Godchau M, Brun P. Anxiety and depression in inpatients. Lancet. 1985;2(8469-8470):1425-1426. doi:10.1016/S0140-6736(85) 92589-9

36. van der Molen T, Willemse BW, Schokker S, Ten Hacken NH, Postma DS, Juniper EF. Development, validity and responsiveness of the Clinical COPD Questionnaire. Health Qual Life Outcomes. 2003;1(1):13. doi:10.1186/1477-7525-1-13

37. Michielsen HJ, De Vries J, Van Heck GL. Psychometric qualities of a brief self-rated fatigue measure: the fatigue assessment scale. J Psychosom Res. 2003;54(4):345-352. doi:10.1016/S0022-3999(02)00392-6

38. Dancey CP, Reidy J. Pearson Education. Stat Without Maths Psychol. 2007;1:245.

39. Delacour H, Servonnet A, Perrot A, Vigezzi JF, Ramirez JM. [ROC (receiver operating characteristics) curve: principles and application in biology]. Ann Biol Clin. 2005;63(2):145-154. Article in French. 
40. Gale NS, Albarrati AM, Munnery MM, et al. Frailty: a global measure of the multisystem impact of COPD. Chron Respir Dis. 2018;15 (4):1479972317752763. doi:10.1177/1479972317752763

41. Paneroni M, Ambrosino N, Simonelli C, Bertacchini L, Venturelli M, Vitacca M. Physical activity in patients with chronic obstructive pulmonary disease on long-term oxygen therapy: a cross-sectional study. Int J Chron Obstruct Pulmon Dis. 2019;14:2815-2823. doi:10.2147/COPD.S228465

42. Blodgett J, Theou O, Kirkland S, Andreou P, Rockwood K. The association between sedentary behaviour, moderate-vigorous physical activity and frailty in NHANES cohorts. Maturitas. 2015;80 (2):187-191. doi:10.1016/j.maturitas.2014.11.010

43. Warburton DE, Bredin SS. Reflections on physical activity and health: what should we recommend? Can J Cardiol. 2016;32 (4):495-504. doi:10.1016/j.cjca.2016.01.024

44. Baltzan MA, Scott AS, Wolkove N, et al. Fatigue in COPD: prevalence and effect on outcomes in pulmonary rehabilitation. Chron Respir Dis. 2011;8(2):119-128. doi:10.1177/1479972310396737

45. Janssen DJ, Spruit MA, Leue C, et al. Symptoms of anxiety and depression in COPD patients entering pulmonary rehabilitation. Chron Respir Dis. 2010;7(3):147-157. doi:10.1177/ 1479972310369285

46. Soysal P, Veronese N, Thompson T, et al. Relationship between depression and frailty in older adults: a systematic review and meta-analysis. Ageing Res Rev. 2017;36:78-87. doi:10.1016/j. arr.2017.03.005
47. Ni Mhaolain AM, Fan CW, Romero-Ortuno R, et al. Frailty, depression, and anxiety in later life. Int Psychogeriatr. 2012;24 (8):1265-1274. doi:10.1017/S1041610211002110

48. Kocks JW, Tuinenga MG, Uil SM, van den Berg JW, Stahl E, van der Molen T. Health status measurement in COPD: the minimal clinically important difference of the clinical COPD questionnaire. Respir Res. 2006;7(1):62. doi:10.1186/1465-9921-7-62

49. Brighton LJ, Evans CJ, Man WDC, Maddocks M. Improving exercise-based interventions for people living with both COPD and frailty: a realist review. Int $J$ Chron Obstruct Pulmon Dis. 2020;15:841-855. doi:10.2147/COPD.S238680

50. Karpman C, Benzo R. Gait speed as a measure of functional status in COPD patients. Int $J$ Chron Obstruct Pulmon Dis. 2014;9:1315-1320. doi:10.2147/COPD.S54481

51. Schoon Y, Bongers K, Van Kempen J, Melis R, Olde Rikkert M. Gait speed as a test for monitoring frailty in community-dwelling older people has the highest diagnostic value compared to step length and chair rise time. Eur J Phys Rehabil Med. 2014;50(6):693-701.

52. Giuliani A. The application of principal component analysis to drug discovery and biomedical data. Drug Discov Today. 2017;22 (7):1069-1076. doi:10.1016/j.drudis.2017.01.005

International Journal of Chronic Obstructive Pulmonary Disease

\section{Publish your work in this journal}

The International Journal of COPD is an international, peer-reviewed journal of therapeutics and pharmacology focusing on concise rapid reporting of clinical studies and reviews in COPD. Special focus is given to the pathophysiological processes underlying the disease, intervention programs, patient focused education, and self management protocols. This journal is indexed on PubMed Central, MedLine and CAS. The manuscript management system is completely online and includes a very quick and fair peer-review system, which is all easy to use. Visit http://www.dovepress.com/testimonials.php to read real quotes from published authors. 